\title{
Towards ubiquitous requirements engineering through recommendations based on context histories
}

\author{
Robson Lima ${ }^{1}$, Alexsandro S Filippetto ${ }^{1}$, Wesllei Heckler ${ }^{\text {Corresp., }}{ }^{1}$, Jorge LV Barbosa ${ }^{1}$, Valderi RQ Leithardt ${ }^{2,3}$ \\ ${ }^{1}$ Applied Computing Graduate Program (PPGCA), Universidade Vale do Rio dos Sinos, São Leopoldo, RS, Brazil \\ 2 VALORIZA, Research Center for Endogenous Resources Valorization, Instituto Politécnico de Portalegre, Portalegre, Portugal \\ 3 COPELABS, Universidade Lusófona de Humanidades e Tecnologias, Lisboa, Portugal \\ Corresponding Author: Wesllei Heckler \\ Email address: weslleiheckler@edu.unisinos.br
}

The growing technological advance is causing constant business changes. The continual uncertainties in project management make requirements engineering essential to ensure the success of projects. The usual exponential increase of stakeholders throughout the project suggests the application of intelligent tools to assist requirements engineers. Therefore, this article proposes Nhatos, a computational model for ubiquitous requirements management that analyses context histories of projects to recommend reusable requirements. The scientific contribution of this study is the use of the similarity analysis of projects through their context histories to generate the requirement recommendations. The implementation of a prototype allowed to evaluate the proposal through a case study based on real scenarios from the industry. One hundred fifty-three software projects from a large bank institution generated context histories used in the recommendations. The experiment demonstrated that the model achieved more than $70 \%$ stakeholder acceptance of the recommendations. 


\title{
Towards Ubiquitous Requirements Engineering Through Recommendations Based on Context Histories
}

\author{
Robson Lima ${ }^{1}$, Alexsandro S. Filippetto ${ }^{1}$, Wesllei F. Heckler ${ }^{1}$, Jorge L. V. \\ Barbosa $^{1}$, and Valderi R. Q. Leithardt ${ }^{2,3}$ \\ $6{ }^{1}$ Applied Computing Graduate Program (PPGCA), University of Vale do Rio dos Sinos \\ (UNISINOS), Av. Unisinos, 950 - Bairro Cristo Rei - CEP: 93.022-750, São Leopoldo, RS, \\ Brazil \\ ${ }^{2}$ VALORIZA, Research Center for Endogenous Resources Valorization, Instituto \\ Politécnico de Portalegre, 7300-555 Portalegre, Portugal \\ ${ }^{3}$ COPELABS, Universidade Lusófona de Humanidades e Tecnologias, 1749-024 Lisboa, \\ Portugal \\ Corresponding author: \\ Wesllei F. Heckler ${ }^{1}$ \\ Email address: weslleiheckler@edu.unisinos.br
}

\begin{abstract}
The growing technological advance is causing constant business changes. The continual uncertainties in project management make requirements engineering essential to ensure the success of projects. The usual exponential increase of stakeholders throughout the project suggests the application of intelligent tools to assist requirements engineers. Therefore, this article proposes Nhatos, a computational model for ubiquitous requirements management that analyses context histories of projects to recommend reusable requirements. The scientific contribution of this study is the use of the similarity analysis of projects through their context histories to generate the requirement recommendations. The implementation of a prototype allowed to evaluate the proposal through a case study based on real scenarios from the industry. One hundred fifty-three software projects from a large bank institution generated context histories used in the recommendations. The experiment demonstrated that the model achieved more than $70 \%$ stakeholder acceptance of the recommendations.
\end{abstract}

\section{INTRODUCTION}

In recent years, the continuous and growing use of new technologies results in a Digital Transformation, bringing disruptive changes across domains (Nadkarni and Prügl, 2021). The techniques considered crucial to eliciting requirements do not hold up, given the paradigm shifts that have occurred. Villela et al. (2019) argued that Requirements Engineering (RE) involves various dimensions and thus ubiquitous RE allows an adequate approach for handling the complexity involved.

The software has become present in the vast majority of businesses, with companies that lack some level of automation being rare. Enterprises need to deal with increasingly diverse, complex, and interconnected systems, while the demand for rapid innovations requires ever-shorter feedback loops. The spread of software in business-to-consumer and business-to-business environments makes it difficult to engage the growing number of stakeholders. Traditional requirements elicitation techniques, such as interviews or focus groups, present problems of scalability and limitation when they need to occur continuously involving the growing number of stakeholders (Villela et al., 2018).

RE stands out as one of the most critical areas for software project results. Factors such as goal setting, project planning, involvement, and identification of user needs are key to project success (Hastie and Wojewoda, 2015). In the meantime, incorrect application of RE is a primary reason for project failures, increasing development time, and cost (Dick et al., 2017; Project Management Institute, 2017b; Bozyiğit et al., 2021). When proper requirements management is applied, the chances of project success increase. 
Studies indicate that $30 \%$ of project success factors are related to RE processes (Hastie and Wojewoda, 2015). Apart from that, reusing requirements can help in the execution of projects, reducing the time for analysis of requirements and identifying reusable code and artifacts, such in case of software development (Irshad et al., 2018).

One option for addressing the issues faced by requirements engineers is requirements reuse. Software Engineering Recommendation Systems (SERSs) help teams select information and make decisions when they are inexperienced or unable to consider all available data. However, setting context is a challenge for recommendation systems (Robillard et al., 2014).

The use of ubiquitous computing (Lopes et al., 2014) is an alternative for assisting requirements engineers in their activities. The classical works of Weiser (1999), Satyanarayanan (2001), and Dey et al. (2001) defined the ubiquitous computing and context-aware computing. Since then, these concepts have been applied in different knowledge areas such in health (Vianna and Barbosa, 2014, 2019; Dias et al., 2020; Petry et al., 2020; Bavaresco et al., 2020), well-being (Vianna et al., 2017), competence management (Rosa et al., 2015), learning (Barbosa et al., 2011; Wagner et al., 2014; Barbosa et al., 2014; Larentis et al., 2020), commerce (Barbosa et al., 2016), accessibility (Tavares et al., 2016; Barbosa et al., 2018), Smart Cities (Rolim et al., 2016; Orrego and Barbosa, 2019; Matos et al., 2021), and agriculture (de Souza et al., 2019; Bhanu et al., 2019; Helfer et al., 2020). The application of ubiquitous computing in project management coined the term Ubiquitous Project Management (Filippetto et al., 2020).

The ubiquitous computing is aware of contexts and allows to use this information to introduce context awareness in the computational systems. Based on contexts, the systems adapt the execution according to the strategic information obtained in the runtime (Abech et al., 2016). Recently, the use of context-aware computing to support the development and maintenance of software emerged as a strategic research theme (D'Avila et al., 2020a,b). In addition, in disruptive applications the ubiquitous computing has been considered an alternative to develop hygge software (Vianna et al., 2017). As a recent evolution, ubiquitous computing has been empowered with the use of temporal series of contexts to organize and analyze the data. This new knowledge research area received the name of Context Histories (Rosa et al., 2015; Martini et al., 2021; Aranda et al., 2021; Machado et al., 2021) or Trails (Silva et al., 2010; Barbosa et al., 2016, 2018). This kind of organization allows the exploration of advance strategies to data analysis, such as, profile management (Wagner et al., 2014; Barbosa et al., 2017; Leithardt et al., 2018; Dalmina et al., 2019; Ferreira et al., 2020; Leithardt et al., 2020), pattern analysis (Dupont et al., 2020), context prediction (Da Rosa et al., 2016), and similarity analysis (Wiedmann et al., 2016; Filippetto et al., 2021).

This article presents a model for recommending requirements in software projects, called Nhatos. The proposed model differs from previous literature in exploring the similarity of project context histories to assist RE processes by predicting future contexts. Thus, new requirements are recommended both in the early stages and throughout the project life cycle. The study seeks to answer the following research questions: (1) Is it possible to use project context histories to infer requirements in the requirements identification phase, considering the characteristics and similarity of the projects? (2) Does stakeholder collaboration, providing project characteristics and feedback from recommendations contribute throughout the requirements management processes?

This article has five sections. The next section discusses related works focusing on the scientific contributions. The third section proposes the model, mainly describing its architecture, the similarity analysis strategy, and the proposed Ontology of Requirements Recommendation. The fourth section describes implementation aspects focusing on prototype characteristics, such as technologies, features, screens, and database model. The section focused on evaluation aspects mainly addresses the application of the prototype in two case studies based on 153 real software projects. Finally, last section presents the conclusion, answers the research questions, and suggests future works.

\section{RELATED WORKS}

The selection of related works demanded the identification of studies that involve the development of models for Requirements Management. The criteria adopted for the choice of works prioritized articles that addressed: (i) models or systems for recommending requirements; (ii) similarity analysis of projects or their requirements; (iii) feedback system on recommendations for new requirements.

Kim et al. (2019) presented an ontology knowledge base and the design process for recommending security requirements based on the cases of attack and the system domain knowledge. The base has three parts: Ontology APT, Ontology of security general knowledge, and Ontology of domain-specific 
knowledge. Each ontology can help in understanding the security concerns in their knowledge. Integrating three ontologies with the problem domain ontology allows the derivation of suitable security requirements with the recommendation process of security requirements. The proposed knowledge base and the process can help to derive the security requirements, considering attacks in real systems.

Liu et al. (2018) conducted a study that approaches the development or maintaining of Android applications. The authors analyzed the multiple challenges that developers face in creating explanations about permissions use. They proposed a new structure, which explores possible security requirements recommendations through the description of similar applications. The study uses techniques of information retrieval and text abstract to find frequent uses of permission.

Xie et al. (2017) proposed a methodology that uses Conditional Random Fields (CRF) to provide a quantitative exploration of the interactions between users and systems in order to discover potential requirements. By analyzing user behavior patterns at runtime, domain experts made predictions about how users' intentions change. The authors proposed improvements to help address the similar needs identified.

Bakar et al. (2016) presented a semi-automated approach, known as Feature Extraction for Reuse of Natural Language Requirements (FENL), for extracting phrases that may represent software resources. The authors aim to extract resources from product reviews online, thus allowing the reuse of software requirements.

Portugal et al. (2017) proposed the use of a software versioning repository (GitHub) as a source of information. To deal with large masses of data and provide access to suitable sources, the authors created project profiles with useful attributes for RE. Afterward, they applied clustering and Natural Language Processing (NLP) to recommend projects by identifying similar keywords in their description.

Williams and Mahmoud (2017) used the social network Twitter as a requirements source to allow a data-driven, interactive and adaptable RE process. The authors performed an analysis with 4,000 tweets from 10 software systems sampled from various application domains. The results revealed that about $50 \%$ of the tweets collected contained useful technical information. In addition, the results showed that text classifiers like Support Vector Machines and Naïve Bayes can be useful in capturing and categorizing tweets technically informative.

Garcia and Paiva (2016) presented a recommendation system that collects the history of using a Web service, relates this information to requirements, and generates reports with recommendations that can increase the quality of this service. The proposed approach aims to provide analytical reports in a language close to the business. The system indicates new workflows, navigation paths, identifies potential resources to remove, and correlates the requirements and the proposed changes, helping to keep the specification of the software requirements up to date.

Hujainah et al. (2021) proposed a technique for prioritizing requirements and thus selecting the requirements to be developed. While not directly recommending, prioritization helps the selection of requirements and supports the process. The authors addressed this task focusing on specific challenges in this area, such as scalability, lack of automation, and excessive time consumption. The study presented a semiautomated scalable prioritization technique using a multi-criteria decision-making method, clustering algorithms, and a binary search tree. The technique aims to mitigate the need for expert involvement in this process and increase efficiency.

Swathine and Sumathi (2021) worked with requirements traceability and based on this information the proposal indicates which requirements must be considered to support the interested parties in the process. This study used a meta-heuristic approach to create a novel traceability system for analyzing systems' functional requirements. The authors aimed to identify traceable links for supporting decision-making, solving the inconsistency problem, and generating quality requirements.

Mougouei and Powers (2021) allowed the selection of requirements considering dependencies and value to be delivered by the requirements. The authors proposed the Dependency-Aware Requirements Selection, an intelligent system that analyzes the value dependencies among requirements, aiming to reduce the risk of value loss. This model considered the user preferences for the requirements, showing promising results in reducing value loss, including when applied in large requirement sets.

Table 1 shows the characteristics adopted in the comparison between Nhatos and related works. The first item (processes) informs which of the RE processes the articles address - validation (V), elicitation (E), specification (S), or management $(\mathrm{M})$. The second item (recommendation) shows the type of item recommended in the study - requirements $(\mathrm{R})$, wrong definitions $(\mathrm{W})$, or projects $(\mathrm{P})$. The third shows the 
strategic path used by the authors for the recommendations. The fourth item refers to the collaboration of interested parties during the recommendation process. Finally, the last two columns present the environment of the model observation and the type of evaluation.

Table 1. Comparison of Related Works

\begin{tabular}{|c|c|c|c|c|c|c|}
\hline Author & Processes & $\begin{array}{l}\text { Recommen } \\
\text { dation }\end{array}$ & Strategy & $\begin{array}{l}\text { Collabo } \\
\text { ration }\end{array}$ & Environment & Type \\
\hline Kim et al. (2019) & $\mathrm{V}^{1}$ & $\mathrm{R}^{5}$ & Ontologies & $\checkmark$ & Academic & Experiment \\
\hline Liu et al. (2018) & $\mathrm{V}$ & $\mathrm{R}$ & Description & $x$ & Academic & Experiment \\
\hline Xie et al. (2017) & $\mathrm{V}$ & $\mathrm{W}^{6}$ & Historic & $\checkmark$ & Academic & Experiment \\
\hline Bakar et al. (2016) & $\mathrm{E}^{2}$ & $\mathrm{R}$ & Description & $x$ & Academic & Experiment \\
\hline Portugal et al. (2017) & $\mathrm{E}$ & $\mathrm{P}^{7}$ & Commits & $x$ & Industry & Use Case \\
\hline Garcia and Paiva (2016) & $\mathrm{E}$ & $\mathrm{R}$ & Logs & $\checkmark$ & Academic & Experiment \\
\hline Hujainah et al. (2021) & $\mathrm{V}$ & $\mathrm{R}$ & Historic & $x$ & Academic & Experiment \\
\hline Swathine and Sumathi (2021) & $\mathrm{V}$ & $\mathrm{W}$ & Historic & $\checkmark$ & Academic & Experiment \\
\hline Mougouei and Powers (2021) & $\mathrm{E}$ & $\mathrm{R}$ & Expert System & $x$ & Academic & Experiment \\
\hline Nhatos Model & $\mathrm{V}, \mathrm{E}, \mathrm{S}^{3}, \mathrm{M}^{4}$ & $\mathrm{R}$ & $\begin{array}{l}\text { Context } \\
\text { Histories }\end{array}$ & $\checkmark$ & Industry & Use Case \\
\hline
\end{tabular}

$\begin{array}{lllllll}{ }^{1} \text { Validation } & { }^{2} \text { Elicitation } & { }^{3} \text { Specification } & { }^{4} \text { Management } & { }^{5} \text { Requirements } & { }^{6} \text { Redefinitions } & { }^{7} \text { Projects }\end{array}$

The analysis of related works indicates four scientific contributions of Nhatos. First, the proposal collaboratively approaches all RE processes, allowing everyone involved to contribute throughout the projects. Nhatos collects different points of view on requirements at any time during the life cycle of the projects, contemplating all RE processes. Second, the model addresses requirements recommendations at the beginning of a new project, using histories of projects already executed, through common characteristics between projects and requirements. Third, the similarity analysis of context histories and NLP allow the recommendation of similar requirements in the initial phase of projects. Finally, Nhatos recommends future contexts based on the similarity analysis of context histories.

\section{PROPOSED MODEL}

According to Robillard et al. (2014), an SERS needs to have specific requirements to be considered a recommendation system, which are: (a) a mechanism for collecting data and artifacts from the development process in a data model; (b) a recommendation mechanism to analyze the data model and generate recommendations; and (c) a user interface to trigger the recommendation cycle and present its results.

Nhatos meets the three requirements mentioned, because: (a) it collects data through a multi-agent system throughout the entire project life cycle; (b) it generates a recommendation considering the current context of the project, and (c) it has an interface on mobile devices to present the results to users and collect their feedback.

In order to measure the applicability of an intelligent tool to support requirements engineers, we conducted a survey involving software design professionals. This research aimed to answer whether the project teams need a proactive tool to support their activities involving the RE processes.

\section{Principles of Nhatos: Survey with 56 Professionals}

A survey involved 56 professionals working in the software development industry, including project managers, analysts, project teams, and teachers. Participants answered an electronic questionnaire with multiple choice and transcribed questions. About $71 \%$ of the interviewees had more than five years of experience in projects. More than $70 \%$ of respondents worked in companies with more than 100 employees. The main objective of the research was to capture the perception of professionals regarding the support tools in project management currently used in their work environment. This research allowed to identify gaps and possible improvements in the RE area guiding the specification of Nhatos. The following are the research questions and results:

- Which areas do you consider most critical to the success of the project? 50\% of respondents selected the scope as the most critical area. Participants also mentioned the areas of Time, Communications, and Integration, with $44.6 \%, 42.9 \%$, and $39.3 \%$ of responses, respectively; 
- In the projects where problems occurred, what were the areas in which the problems were identified? A total of $39.29 \%$ of the participants answered that problems in project management are due to incorrect Scope Management (SM). Other project areas, such as Time Management and Communications, obtained $25(44.6 \%)$ and 21 (37.5\%) responses, respectively;

- What types of suggestions would you like to receive from a proactive project management tool? According to the interviewees' perception, $32.1 \%$ answered that a tool should suggest new requirements for projects;

- Do you believe that information from other projects already completed could assist in project management? $85.7 \%$ of the members confirmed that history contributes to the management of the new projects.

The perception of the teams collected in the survey allowed to conclude that there is interest from the professionals regarding the use of an intelligent tool to support the project teams during the RE processes. This opportunity stimulated the development of the Nhatos, which aims to assist teams during the RE process life cycle.

\section{Model Architecture}

Fig. 1 shows Nhatos architecture using the Technical Architecture Module (TAM) modeling specification (SAP, 2021). The following components are part of the model and they are seen in the figure with their respective numbering:
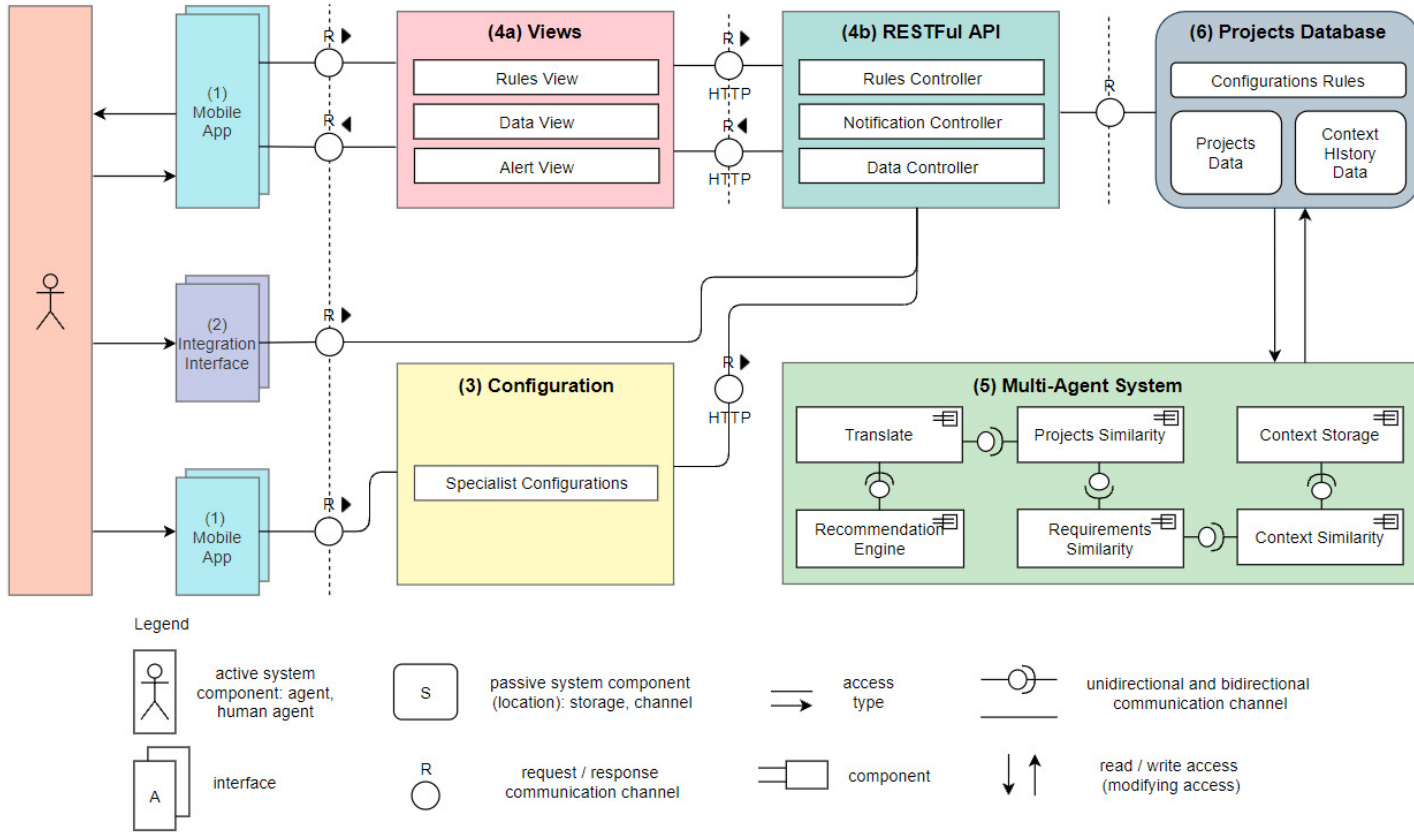

Figure 1. Nhatos Architecture

1. Mobile App: Application in a hybrid structure based on Javascript language, which operates both in mobile applications (Android / IOS), as well as in browsers web. Stakeholders use this application during interactions in RE processes. The stakeholders can: (a) add and manage projects and their information; (b) add requirements and modify them; (c) view the recommendations generated by the model; (d) provide feedback about the recommendations received, accepting or rejecting them;

2. Integration Interface: It enables the bulk import of data from ongoing projects. When using this system web interface, users may import projects and requirements exported from third-party project management tools, such as MS Project, for example; 
3. Configuration: Project team preferences are entered through an assistant agent, accessed via mobile devices. Each project variable receives a weight (area of knowledge, size, methodology, and level of completeness of the schedule). Each recommendation made by the model considers the weight configured by a specialist.

4. Views and API: These applications operate in an integrated manner in a server environment.

(a) Views: They are characterized by controllers responsible for the business rules of the model, obtaining information already stored in the database to provide information to those involved;

(b) API: A data access interface which uses the RESTFul protocol to interconnect the application Mobile App and Database.

5. Agents: Multi-Agent System (MAS) that captures the events related to project's evolution or modification. The capture is triggered when some of these events occur: (a) addition of a new requirement; (b) termination of an activity; or (c) evolution in the percentage of completion of the project. Fig. 2 shows the proposed MAS using the the Prometheus methodology (Larioui, 2020). The MAS has six agents. The Translate agent converts to English the texts from native languages used in the projects. The NLP uses English as the language, so this translation is necessary to Nhatos. Projects Similarity analyzes the similarity of the projects using project size, methodology applied and area of expertise. NLP techniques allow to group projects according to their expertise. Context Storage stores each event occurrence in the project's history. Recommendation Engine permanently monitors the project's events to orchestrate the execution of the other agents when one event occurs. Requirements Similarity uses semantic analysis to determine the requirements similarity based on texts written in natural language. This analysis is detailed in the Similarity Analysis subsection. The agent also compares requirements to determine if requirements have the same number of actors. Context Similarity performs the similarity analysis in the context histories of the projects.

6. Projects Database: It saves application settings, such as (a) Project data; (b) Recommendations made by the model; (c) Feedback from stakeholders regarding the recommendations; and (d) Context histories that occurred throughout the life cycle of the projects.

\section{Similarity Analysis}

The similarity analysis occurs in two moments: (1) similarity analysis based on project characteristics, and (2) similarity analysis based on context histories of projects. The first analysis occurs at each insertion of a new project, while the second analysis occurs during a new evolution of the projects' life cycle. Fig. 2 shows the representation of the multi-agent system with the three agents that conduct the similarity analysis (Context, Projects and Requirements).

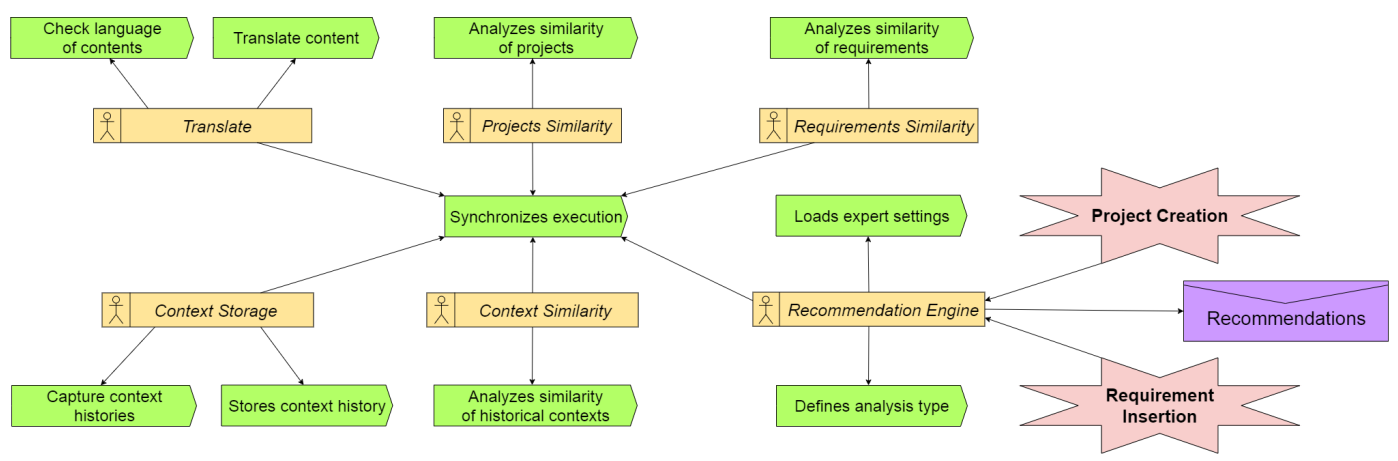

Figure 2. Multi-Agent System

The model captures the characteristics of the new project during its creation. At this time, the project teams have not yet implemented it. The project is in the creation or planning phase. Project Management Institute (2017a) has characteristics that are considered properties of a project. Stakeholders 
determine these characteristics in the early stages of the life cycle, which are: (a) Area of knowledge; (b) Development methodology (agile, traditional, or hybrid); (c) Level of completeness of the schedule; and (d) Size. Fig. 3 shows how the recommendation flow occurs in this step.

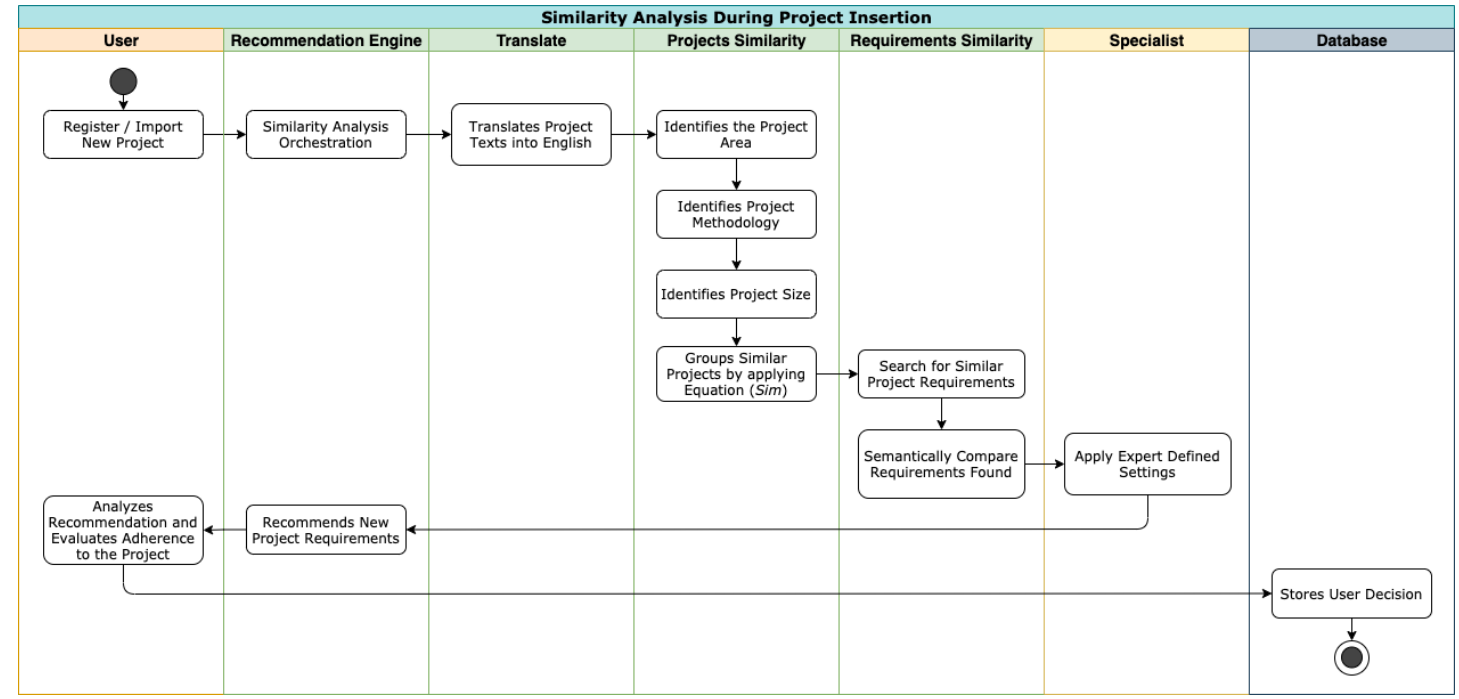

Figure 3. Similarity Analysis by Project Characteristics

After capturing the fundamental characteristics of the project, an expert defines the weights for each one. The specialist defines the importance of each characteristic, based on its degree of relevance to the project and organization. This definition allows to measure the similarity between the projects before the start of their execution.

After the insertion of a new project, the multi-agent system identifies this event and initiates the recommendation process. The multi-agent system goes through the stored histories and compares the variables with characteristic of each project in the history with the same variables as the original project. The model considers the configurations previously informed by the specialist - these configurations make up a weight system, which will be applied during the calculation.

After the model groups similar projects, the context histories of those projects are analyzed to identify reusable requirements among them. Therefore, for projects in the same group, Nhatos calculates similarity considering the semantic distance between their requirements.

Nhatos defines the semantic distance by analyzing the distance between text documents proposed by Kusner et al. (2015). This approach takes advantage of the results of Mikolov et al. (2013), whose model word2vec generates combinations of words, on a large-scale ontology, for extensive data sets (for example, we use the training of approximately 100 billion words in this model). In this way, Nhatos compares the texts that describe the objectives of the requirements. Afterward, the results are stored in history, enabling the recommendation of requirements related to the same theme (similar purposes) and similar projects (same area of knowledge).

The recommendation of requirements in the initial phases of the projects aims to bring historical information to the project teams, mainly to the requirements engineers and stakeholders. Then, these users will be able to accept or reject the requirements recommended by the model. Nhatos thus ensures that no requirements of the historical basis are disregarded by those involved during management.

During the life cycle of projects, Nhatos saves the events in context histories. The model identifies information that is susceptible to changes in state over the life cycle of the projects, which are: (a) purpose of the requirements; (b) actors involved in the requirements.

Whenever a user inserts a new requirement into the project or at least one of this context information is modified, the similarity analysis of projects by context histories begins. Nhatos uses the stored histories to complement the similarity analysis based on the characteristics, updating recommendations based on the new information.

Fig. 4 shows how the recommendation flow occurs in this step. This flow seeks similar contexts by analyzing the context histories of the project and comparing this information with other stored contexts. 


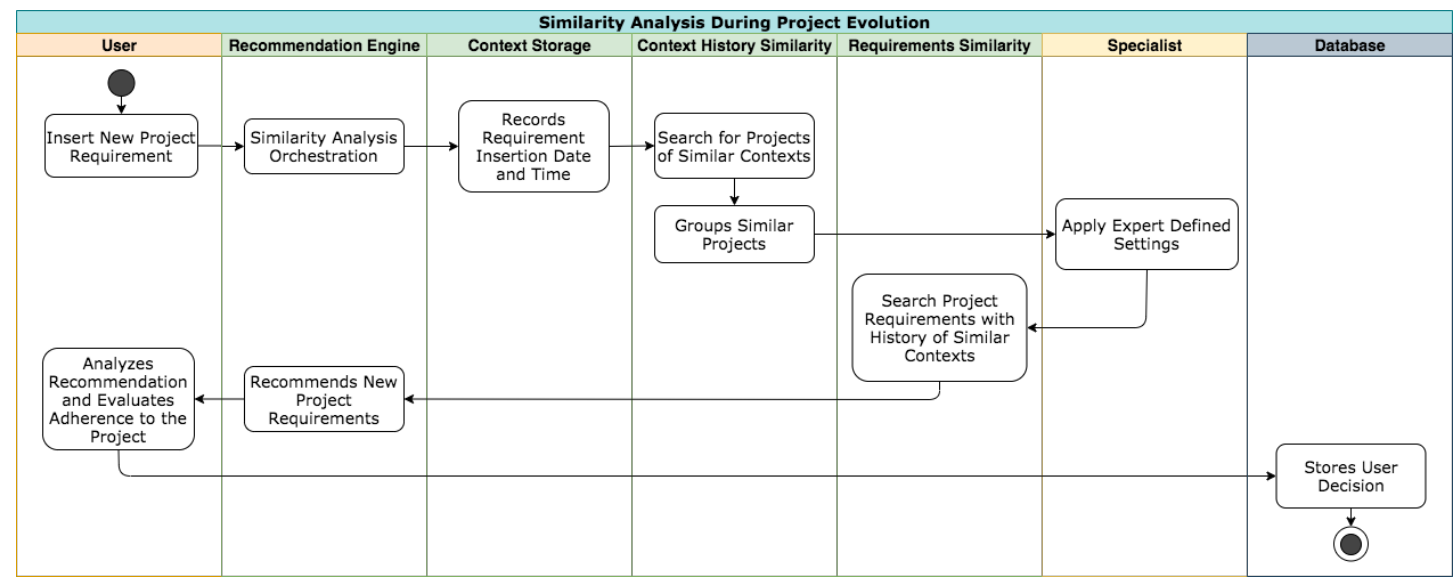

Figure 4. Similarity Analysis by Project Context Histories

Each step presented in the projects' timeline represents information about event occurrences saved in context histories. In this way, this information can later be used by the model to generate a new recommendation. Whenever one of the defined events occurs, Nhatos creates a record in the context history of the project.

Nhatos compares each project context with contexts from similar projects by using the semantic distance between the requirements from the previously-stored histories. The recommendation of the next occurrence of the context history occurs for the project in execution when the distance between the requirements is acceptable (according to the specialist's settings), and the number of actors is equal between the requirements compared.

Fig. 5 shows an example of analyzing the context histories of an ongoing project with histories from similar projects. In this example, the entire recommendation flow is elucidated step by step.

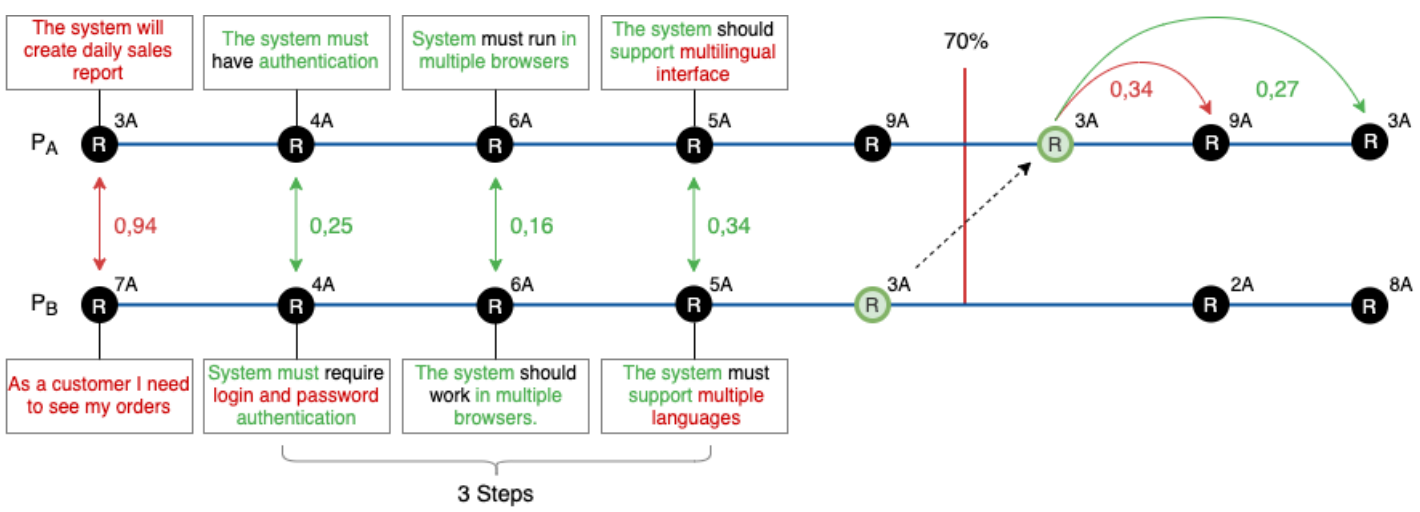

Legend

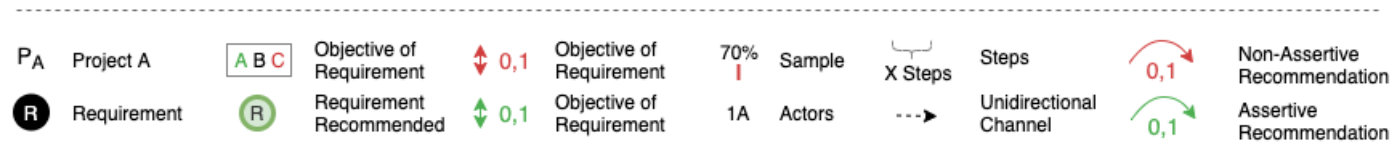

Figure 5. Similarity Analysis by Context Histories

We considered two projects to exemplify the similarity analysis of context histories. Both projects ( $\mathrm{Pa}$ and $\mathrm{Pb}$ ) have five requirements. The multi-agent system compares requirements between projects in chronological order. In this scenario, the specialist configured the minimum semantic distance of 0.3 , training sample $70 \%$, and three similar steps to generate the recommendations. Thus, the comparison of the first requirement of Project A with the first requirement of Project B generated a semantic distance of 0.41 (distance not acceptable according to the configured parameters). Another excluding factor is that these requirements have a different number of actors. Thus, step 1 is not similar. On the other hand, 
steps 2,3 , and 4 obey both the minimum acceptable semantic distance and the same number of actors involved in the compared requirements. Since the expert has configured recommendations that require at least three similar consecutive steps, Nhatos recommends the fifth requirement of project B for project A.

Assuming that the configured training sample was $70 \%$, Nhatos uses the remaining percentage of the project (30\%) to verify that at least one requirement with the same semantic distance and the same number of actors as the recommended requirement occurred throughout the life cycle. Once it occurs, the recommendation made is considered assertive.

The analysis of more than one chronological context, which occurred during the project, aims to identify projects that have a similar execution sequence. This analysis contributes to a higher degree of precision in the recommendations made. The most significant number of similar consecutive contexts indicates the proximity between project implementation. However, when Nhatos considers more contexts, the fewer projects must be identified as similar and, therefore, the fewer recommendations made, since each project is unique (Project Management Institute, 2017a).

\section{Ontology of Requirements Recommendation}

Fig. 6 shows the Ontology of Requirements Recommendation proposed by Nhatos. The domain ontology contains Projects, Requirements, and Specification. This representation is an extension of the work of Silver (2014), with the addition of the Projects Ontology. Three ontologies covered the domain considered by Nhatos: a) Requirements Specification Ontology which makes up the requirements specification; b) Projects Ontology which is characterized by the domain of the project and its contextual information; and c) Requirements Ontology that represents the requirements.

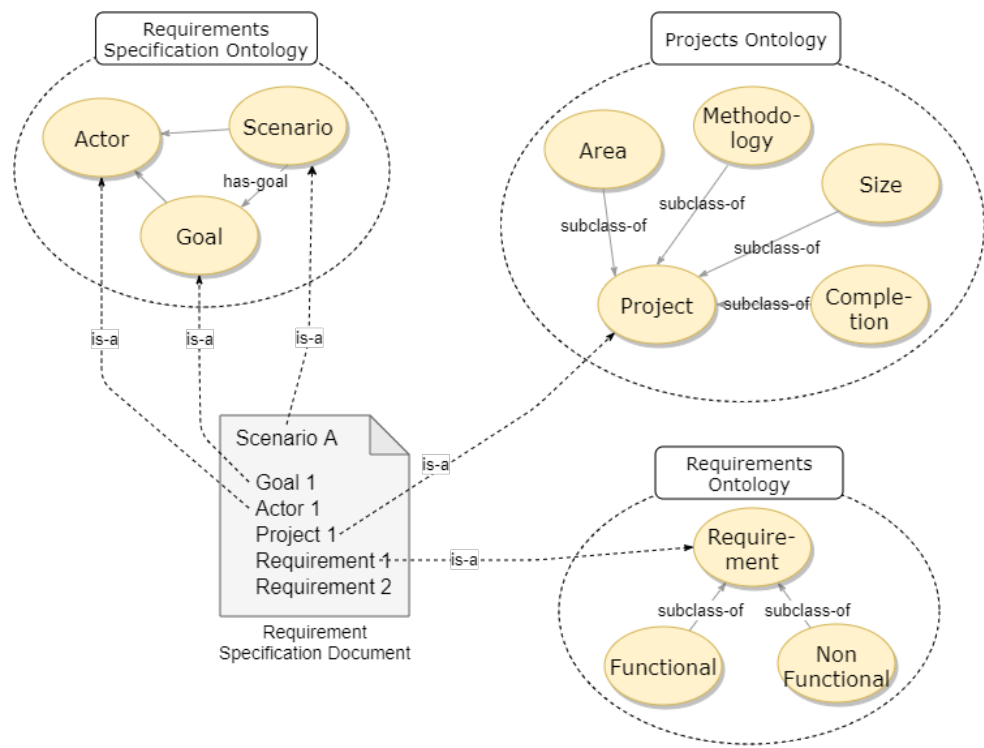

Figure 6. Ontology of Requirements Recommendation

The project ontology has the Project class composed of the Area, Completion Level, Methodology and Size. These subclasses define the contextual information of the projects. The ontology that comprises the requirements specification has the classes Scenario, Goal, and Actor. These classes represent the elicitation process, identifying new requirements and relating them to projects. The ontology that determines the requirements has the class Requirement and its subclasses Functional Requirement and Non Functional Requirement, which define the types of requirements.

We added a specification document (Requirement Specification Document) to exemplify how the requirement is instantiated when using ontologies. Each document has a scenario, where the actors through the defined objectives identify requirements for a given project. 


\section{IMPLEMENTATION ASPECTS}

We developed a software prototype to meet the model definitions. Fig. 7 shows that the prototype integrates three applications: (1) Console Application ${ }^{1}$; (2) RESTFul API Application or WebService ${ }^{2}$; and (3) Hybrid Application ${ }^{3}$, composed by the Web Application and the Mobile App. The first two are back-end applications, which run on a server. The third application operates on mobile devices, acting as front-end software. The users involved in the requirements engineering processes used this application.

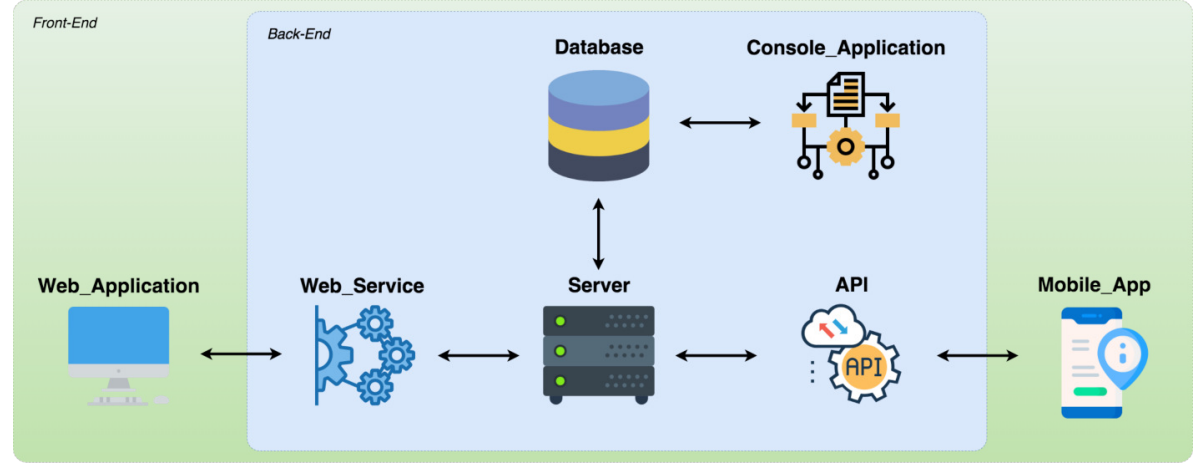

Figure 7. Prototype Overview

\section{Hybrid Application}

The Hybrid Application runs both on mobile devices and on conventional computers operating in a browser web. In this way, the application has two interfaces for communication with system users: (1) Web Application; and (2) Mobile App.

This application allows the interaction of project teams with Nhatos, allowing the management of projects, requirements, activities, resources, registration of interested parties, and evaluation of recommendations. The prototype allows to monitor projects, capturing context information to compose the context histories. The software can be used throughout the life cycle of projects. Further, this application presents the recommendations to the user, thus allowing the collection of feedback from interested parties.

Fig. 8a shows the interface for the project presentation with characteristic information about the project such as size, methodology, percentage of evolution, and area of knowledge. Fig. $8 \mathrm{~b}$ presents the list of project requirements, as well as their respective percentage of evolution.

Fig. 9a shows the settings of the weight variables, which are defined by an expert. These variables define the importance of each aspect of the project and its requirements during the recommendation process. The user can define weights related to project area, size, methodology, and level of completion, as well as the acceptable semantic proximity between the requirements that will be considered for a possible recommendation.

Fig. $9 \mathrm{~b}$ presents examples of recommendations. The interface contains the recommended requirement, as well as the requirement that raised this recommendation and the semantic distance between these requirements. The interaction area also enables users to provide their feedback, selected from the options to accept or reject the recommendation. This user decision is registered in order to evaluate the acceptability of the recommendations by stakeholders in the future.

\section{Console Application}

This application developed in Python is an encapsulated software that acts in the form of service. The software uses the concept of multi-agent systems, proposed by Padgham and Winikoff (2004) to implement the Agents layer. The application does not depend on direct interaction with the users, being triggered by modifications detected in the environment. The Agents layer implements the following 6

\footnotetext{
${ }^{1}$ https://github.com/robsonklima/nathos-py-v2

${ }^{2}$ https://github.com/robsonklima/nhatos_api

${ }^{3}$ https://github.com/robsonklima/nhatos_front_end
} 


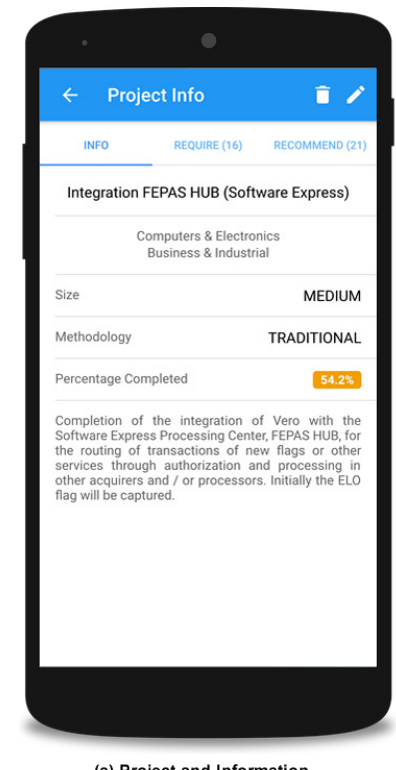

(a) Project and Information

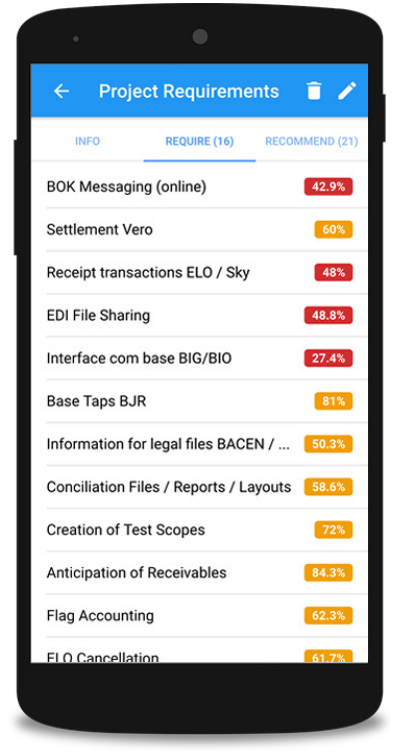

(b) Requirements and Percentage of Activities

Figure 8. Screenshots with Project Details and Requirements

agents: (1) Recommendation Engine; (2) Translate; (3) Projects Similarity; (4) Requirements Similarity; (5) Context Similarity; and (6) Context Storage.

The Recommendation Engine runs systematically through the detection of changes occurred in the environment. The agent always activates when a user inserts a new project or requirement. Once performed, this agent is responsible for starting the execution of all other agents.

The Translate agent translates all the contents entered by the user into the English language, enabling the execution of subsequent agents. Since, as a premise, all contents must be registered in English. Nhatos uses NLP and the corpus of texts obtained for the application of the study is written in this language. The software loads the projects and their requirements from the database, translating them using the API Google Cloud Translate (Google, 2021b). We considered the usage of this API does not generate discrepancies in translations since this API has an estimated accuracy of 85\% (Aiken, 2019) and the texts translated are technical, having a technical writing pattern.

The Projects Similarity agent activates after the translation of the content of the projects and their requirements. This agent analyzes the similarity between all projects in the database and groups the characteristics of the projects separately. The software considers the information in the ontology (Fig. 6), considering all projects in the database according to size, area of knowledge, management methodology, and level of completeness (schedule). After consulting all projects, the agent classifies and labels each project, so that the next step of the algorithm starts.

The Requirements Similarity employs the use of NLP to find requirements that contain equivalent objectives, as well as the same number of actors involved. This agent also considers the similarity analysis between the projects, performed previously. In this way, the algorithm analyzes the similarity between the requirements of projects considered similar.

First, the agent appropriates the new settings for distance, steps, and sampling, thus starting new processing. Then, it removes the previous recommendations (if any), to start a new recommendation process. This method removes all recommendations that originated from the same distance, number of steps, and sampling setting. Because, it is considered that throughout the life cycle, the requirements may have changed regarding their objectives or actors involved (Project Management Institute, 2017a).

Then, the agent retrieves all projects from the database. The requirements of each project are obtained. Each requirement is compared with the requirements of similar projects. In this step, the agent checks whether the objective of both requirements meets the established distance parameters, as well as the number of actors. Finally, once the number of requirements found sequentially is equal to the number of configured steps, this requirement is considered recommendable to the project.

The Context Similarity agent analyzes whether the recommendations carried out within the sample 


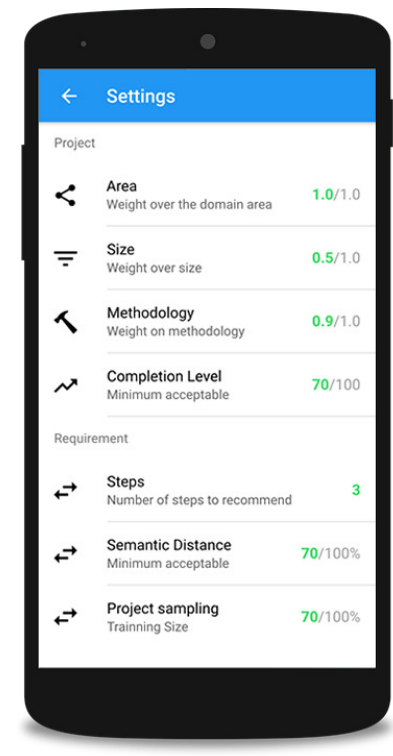

(a) Configurations

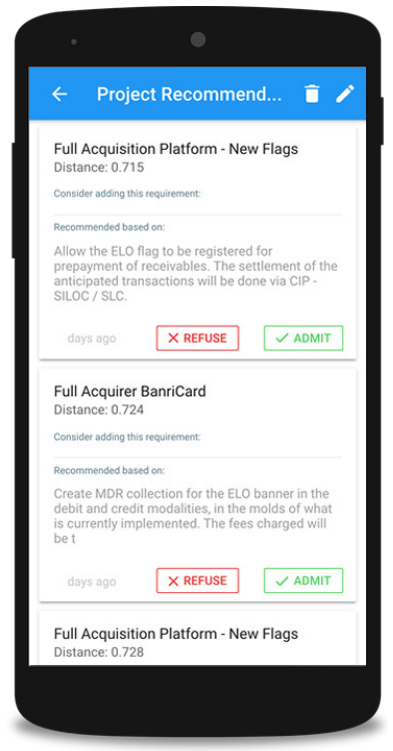

(b) Evaluation of Recommendations

Figure 9. Screenshots with Specialist Settings

selected by the specialist occurred in the project. The software loads all recommendations made and it selects the project requirements after the recommendation's record date. If among the requirements, a requirement is found with the same semantic distance to the compared requirement and, containing the same number of actors, this recommendation is considered assertive. Otherwise, the recommendation becomes non-assertive. Finally, this decision is stored in the database.

The Context Storage agent keeps the information in a database with four main entities: (1) projects; (2) requirements; (3) requirements distance; and (4) recommendations.

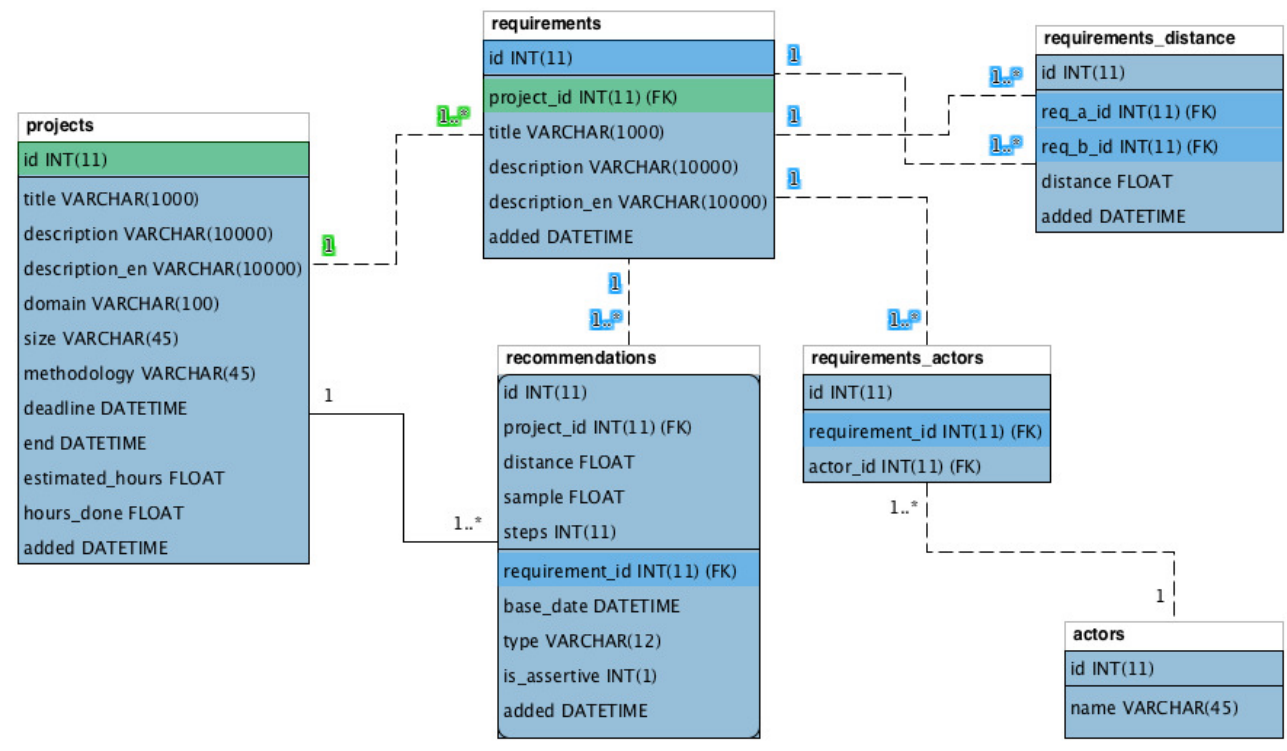

Figure 10. Relational Entity of the Nhatos Model

Fig. 10 shows the relational entity diagram of the model database. The projects entity saves information related to projects. This entity registers basic project information and its characteristic information, such as: opening statement (description), knowledge area (domain), size (size), and methodology applied during its development (methodology). 
The requirements entity keeps model requirements. The same project can contain several requirements, according to the relationship in the diagram (1..n). The actors of each requirement are stored in the entity actors and an actor can be linked to several requirements and vice versa, as shown in the entity requirements_actors $(\mathrm{n} . . \mathrm{n})$.

The requirements_distance entity saves the distance information between the processed requirements. The entity stores the original requirement (req_a_id) and the compared requirement (req_b_id), as well as the respective semantic distance (distance) between them.

Finally, the recommendations entity keeps the recommendations inferred by Nhatos. Each recommendation is directed to a project (project_id) and the requirement that generated such a recommendation (requirement_id). The assessment of the assertiveness of each recommendation is stored in the is_assertive property.

\section{RESTFull API Application or Web Service}

The RESTFull API Application provides a communication channel between the Hybrid Application and the Context Storage application. Using the RESTFull protocol, the application allows data traffic in the JSON format between applications. It enables the exchange of data between the hybrid application, used by users, and the information already processed by Console Application, which stores its information in the Nhatos model database.

\section{EVALUATION ASPECTS}

The application of a case study in a software development company allows answering the research questions. This company develops application solutions for a banking institution. This study aimed to confirm the hypothesis of using the analysis of context histories of projects to recommend requirements for new or ongoing projects.

The study employed a database with the context histories of 153 software development projects. The database has projects with different resources and development methods, such as distributed or local teams. The use of different characteristics of the 153 projects allowed the analysis of a diversity of contexts to the recommendation of requirements.

The evaluation considered two cases: (1) two teams evaluated the use of the prototype during the implementation of 5 real projects, and (2) 17 completed projects were used to evaluate the recommendations made by Nhatos, comparing the recommendations done with the requirements in the 17 original projects. Next subsections describe these evaluations.

\section{Team Evaluation During Project Execution}

The first case involved two teams with a total of 12 professionals. These professionals were asked to validate the recommendations made by the model. Table 2 shows the profile of the teams that participated in this experiment.

Table 2. Profile of Participating Teams

\begin{tabular}{l|c|c|c}
\hline Team & Role & Experience (Years) & Mode \\
\hline Team A & Scrum Master & $15+$ & Local \\
\hline & Product Owner & $10+$ & Distributed \\
\hline & Designer & $5+$ & Local \\
\hline & Developer & $10+$ & Local \\
\hline & Developer & $5+$ & Local \\
\hline & Developer & $5+$ & Local \\
\hline & Test Analyst & $5+$ & Local \\
\hline Team B & Project Manager & $25+$ & Distributed \\
\hline & Developer & $10+$ & Distributed \\
\hline & Developer & $5+$ & Local \\
\hline & Developer & $5+$ & Local \\
\hline & Test Analyst & $5+$ & Local \\
\hline
\end{tabular}

Initially, project teams inserted information related to the projects in the database (scope and descriptive information, terms of reference, resources, schedule, and tasks/activities). The professionals used the 
integration interface to insert information into the database. After, the project teams identified and registered the requirements using the prototype running on mobile devices.

The similarity analysis of the projects used NLP since approximately $79 \%$ of the requirements and project documents are written in natural language (Luisa et al., 2004). The algorithm classified each project through the use of NLP according to its respective area of knowledge. The identification of these areas involved the use of the opening statement. In addition, the project charter contains a high-level description of the project. The classification used the Google Natural Language API (GNL), which provides resources for the analysis of unstructured texts, such as content classification and entity identification. The content rating analyzes a document and results in a list of categories that apply to the found text. The classification can still contain several levels, specifying the greater depth of details about the area of knowledge in question (Google, 2021a).

Currently, GNL processes English sentences only. The projects had information in Portuguese, so it was necessary to previously translate the descriptive content of the projects before carrying out the classification process. The prototype performed the translation automatically using the Google Cloud Translation API (GCT) (Google, 2021b). The GCT receives a phrase as input, identifies its language and translates it into the language selected by the user. During the study, the agent translated all sentences into English. After translation, Nhatos categorized the projects using GNL based on the 153 projects. This step classified 23 projects as Finance (15.03\%), 20 as Business and Industry (13.07\%), 10 as Computers and Electronics (6.53\%), 6 as Credit and Lending (3.92\%), and 4 as Accounting and Auditing (2.61\%), having these categories the highest number of classified projects.

In addition, the variables registered in the Configuration module allowed to defining the knowledge area of each project. The similarity analysis used this definition. These variables received weights, which reflect the uniqueness of each project. The weight setting allows the algorithm to generate different recommendations according to the characteristics recorded by the specialist. Table 3 presents the recommended requirement values for each project and the requirement values added to new projects through the recommendations.

Table 3. Recommendations Made by Project

\begin{tabular}{l|c|c|c|c}
\hline Project & Area & Recommendations & Accepted & \% Accepted \\
\hline Renegotiated Operations - Restructured & Finance & 16 & 11 & 68.7 \\
\hline Alteração Renov Autom Cheque Especial PF & Finance & 14 & 8 & 57.1 \\
\hline CDB movements in M-BANKING & Business \& Industrial & 9 & 7 & 77.7 \\
\hline Parameterization of Indexers & Finance & 10 & 9 & 90.0 \\
\hline Automatic lock renewal & Finance & 13 & 8 & 61.6 \\
\hline Approval Percent & \multicolumn{4}{l}{} \\
\hline
\end{tabular}

During the case study, users included in the projects twenty requirements, which were used to evaluate the recommendations made in the execution of the project. Whenever a user added a new requirement, the Requirements Similarity Agent identifies the event and performs the semantic proximity analysis. The Agent compares the new requirement description with the requirements stored in the context history of the project.

The GNL algorithm analyzes texts in English, performing a semantic analysis. Thus, the model translates the description of the project or requirements which are in another language. Table 4 shows an example of a requirement added to a project and the recommended requirements based on this insertion. Nhatos considers the objective of each requirement and also the number of related actors.

Table 4. Semantic Analysis for Requirements Recommendation

\begin{tabular}{llc}
\hline Included Requirement & Recommended Requirements & Distance \\
\hline The software must allow the manager & $\begin{array}{l}\text { The software must allow the manager } \\
\text { to request airline tickets ( } 1 \text { actor). }\end{array}$ & 0.21 \\
\cline { 2 - 3 } & $\begin{array}{l}\text { The software must allow the manager } \\
\text { to request resource transfers between } \\
\text { projects }(1 \text { actor). }\end{array}$ & 0.32 \\
\hline
\end{tabular}

At this stage, Nhatos applies the analysis to the entire database, regardless of whether projects were 
compared in the first stage of the recommendation. The semantics of the included text and the objectives of the requirements may change throughout the project (Dick et al., 2017). This step also allows project requirements that were not originally recommended to be analyzed and considered. The analysis is carried out at this point on the requirements, considering the grouping of projects based on their characteristics. The semantic distance represents the comparison of the requirement objectives, having a floating-point value between 0.0 and 1.0. The distance closer to 0 indicates that the recommended requirement is semantically closer to the original.

Consequently, the closer the semantic distance to 1 , the less similar the recommended requirement is considered when compared to the original. The actors of each requirement are also considered during this stage of the analysis. This context information considers the number of actors to which a requirement is related.

During the case study, for each of the five projects, the model analyzed the similarity, recommending the requirements between similar projects. Soon after, the team analyzed the recommended requirements for the five registered projects. Table 3 shows that the approval rate of the requirements had an average of $71.0 \%$. The average appropriation of the recommendations presented for new projects shows the acceptance of the requirement recommendation model evaluated by the teams, in order to provide more information to the managers since the beginning of the project.

\section{Evaluation of Recommendations Through Analysis of Context Histories}

The second case compared the requirements registered in 147 completed projects with the requirements recommendations made by Nhatos. This study allowed to infer whether the recommendations made, considering a sample of $70 \%$ of the progress of the projects, were in fact inserted into the remaining percentage of the project.

This scenario evaluated the recommendations made in many situations considering projects with different characteristics. Most of the projects (81) used the agile methodology based on the framework SCRUM (Sutherland and Coplien, 2019). The others (66) employed traditional methodology based on the good practices proposed by Project Management Institute (2017a). We classified the projects into one of three size categories, considering the execution time of each one: small (up to 500 hours), medium (up to 3,000 hours), and large (over 3,000 hours).

The learning phase used a sample of $70 \%$ referring to the execution of each project. The Nhatos Learning consists of collecting the project's evolutionary events. Figure 4 shows this process in the Recommendation Engine, Context Storage, and Context History Similarity steps. With this learning, Nhatos generated requirement recommendations for the same projects. The remaining $30 \%$ of data from the executed process was the base for assessing the recommendations made by the model.

The similarity analysis between the projects taking into account the context histories considers that a consecutive sequence of contexts must be similar. The model then recommends the next requirements for the project being implemented based on the sequence of events that generated context histories.

In all, 9 test scenarios were configured using different parameterizations to find the best scenario of recommendations, where each test considered the entire historical database. Table 5 shows the results of the different settings applied. A test scenario addresses different combinations of the variables Distance, Steps and Sample.

Table 5. Evaluation of Recommendations Made by the Nhatos

\begin{tabular}{c|c|c|c|c|c|c|c|c}
\hline$\#$ & Distance & Steps & Sample & $\begin{array}{c}\text { Non-Assertive } \\
\text { Recommendations }\end{array}$ & $\begin{array}{c}\text { Non-Assertive } \\
\text { Recommendations (\%) }\end{array}$ & $\begin{array}{c}\text { Assertive } \\
\text { Recommendations }\end{array}$ & $\begin{array}{c}\text { Assertive } \\
\text { Recommendations (\%) }\end{array}$ & Total \\
\hline 1 & 0,25 & 3 & 0,7 & 248 & 51,56 & 233 & 48,44 \\
\hline 2 & 0,25 & 4 & 0,7 & 19 & 70,37 & 8 & 481 \\
\hline 3 & 0,25 & 5 & 0,7 & 1 & 50 & 1 & 29,63 \\
\hline 4 & 0,3 & 3 & 0,7 & 555 & 29,65 & 1317 & 70 \\
\hline 5 & 0,3 & 4 & 0,7 & 194 & 30,03 & 452 & 70,35 \\
\hline 6 & 0,3 & 5 & 0,7 & 56 & 28,43 & 141 & 69,97 \\
\hline 7 & 0,35 & 3 & 0,7 & 904 & 16,96 & 4427 & 71,57 \\
\hline 8 & 0,35 & 4 & 0,7 & 443 & 17,51 & 2087 & 83,04 \\
\hline 9 & 0,35 & 5 & 0,7 & 237 & 17,52 & 1116 & 57 \\
\hline
\end{tabular}

All test scenarios considered a 0.7 sample value. Therefore, the training sample to generate the recommendations contained $70 \%$ of the evolution of the projects' schedule (life cycle). Tests 1, 2 and 3 
considered configurations with a minimum semantic distance between the requirements of $0.25(75 \%)$ All 3 step configurations for these tests resulted in an assertiveness rate equal to or less than $50 \%$. Scenario 2 and 3 generated a few recommendations, 27 and 2, respectively. Test 1 generated a total of 481 recommendations. However, as mentioned, the assertiveness did not reach $50 \%$.

On the other hand, tests 7, 8, and 9 generated a significant amount of recommendations. The assertiveness rate was high, above $80 \%$ in the three cases. However, the semantic distance proved to be relatively comprehensive, considering requirements only $65 \%$ similar. Therefore, the tests allowed the inference of a large number of requirements recommendations (a total of 9,214). In these scenarios, Nhatos provided a high number of recommendations, being more than the teams could assess. Therefore, the model did not attend to a requirement of ubiquitous applications in these cases since these applications must be minimally intrusive (Satyanarayanan, 2001).

Scenarios 4, 5, and 6 were more promising than the first ones. All three achieved a percentage rate of assertiveness close to or higher than $70 \%$. However, only scenarios 4 and 5 had an adequate number of recommendations for analysis in the use case.

The configuration of the Scenario 4 considered a minimum distance of $0.3(70 \%)$ and three steps, obtaining a hit rate of $70.35 \%$ for assertive recommendations. Of the 1,872 inferred recommendations, 1,317 were correct, while 555 were incorrect. Scenario 5 considered a configuration of a minimum distance of $0.3(70 \%)$ and four steps. This scenario obtained a hit rate of $69.97 \%$ of assertive recommendations. 452 of the 646 recommendations were correct, while 194 were unsuccessful. The different scenarios allowed a new round of tests to be carried out, obtaining the most certain configuration for the database.

\section{CONCLUSION}

This article proposed Nhatos, a computational model which provides recommendations considering the characteristics of each new project. In this way, the teams start the life cycle of each project with a broader set of information, making the project planning more assertive, which increases the chances of success.

In addition, Nhatos infers new recommendations during the execution of the projects through the analysis of the included requirements. The recommendations benefited from a semantic analysis of the text that understood the requirements' objectives, as well as the number of actors involved. In this sense, new scenarios for the projects are considered during their implementation. The model considers context histories of projects when recommending new requirements, when considering the schedule of similar contexts and when compared to the original projects.

The research questions allow to validate the use of the Nhatos in two dimensions: (i) requirement recommendation considering the context histories of the projects; (ii) elicitation and specification of requirements, allowing their use collaboratively. In this sense, the results demonstrated the adherence of the Nhatos to proactive requirements management in projects. A summary of the main conclusions is as follows:

1. Nhatos achieved an accuracy of $65.33 \%$ regarding the average value of the 9 test scenarios performed and scenario 7 reached an accuracy of $83.04 \%$.

2. The first research question focused on the suitability of the recommendations made by the model for the new project, considering the team that developed the projects and the projects already executed. The evaluation confirmed the relevance of projects ' context histories for recommending requirements for the projects. Case 1 presented an average recommendation approval rate of $71.0 \%$, proving that Nhatos can make suitable recommendations based on experts' configurations and characteristics of other projects. Case 2 proved as true the hypothesis of using the context histories for the requirements recommendation, achieving a value higher than $80 \%$ of assertiveness in different scenarios through the similarity analysis of the project context histories.

3. The second research question assessed the ability of the model in recommending requirements throughout the projects' life cycle in a collaborative manner. During the project's follow-up period in the case study, the registration of 20 new requirements occurred, in addition to the requirements recommended by Nhatos. All team members participated in a collaborative analysis of each requirement and contributed knowledge during the elicitation, specification, and validation processes, providing more information for the project. The collaboration of the requirements management team allowed the evaluation of possible impacts that may occur in the projects. Also, 
teams could collaborate during all requirements management processes through the use of the prototype.

4. The answers to the research questions confirm the main scientific contributions of this study, which is the recommendation of requirements considering the characteristics of the projects and analyzing the context histories, in addition to monitoring the entire life cycle of the requirements throughout the project. Thus, the model helps in planning projects by providing a broader set of information adhering to the project in progress for requirements engineers when starting a new project.

5. The collaboration of all interested parties enhanced the model, mainly in the identification and specification of requirements. This is not present in the related works. This differential enabled the collection of more information during the implementation of the project and brought technical and practical knowledge about the importance of the requirements by all stakeholders.

6. The case studies and the prototype allowed the evaluation of the Nhatos, contributing to the observations of gaps in the management of project requirements. The case studies focused on answer the two research questions presented in the introduction.

Based on the results obtained in the case studies, we suggest the following opportunities that future studies can explore:

1. The exploration of the model usage, considering projects from different companies.

2. The monitoring of the prototype use over time since the prototype can provide a more robust design history and more assertive requirement recommendations, considering the model benefits from a growing database.

3. The exploration of the pattern analysis of context histories, which can allow detecting emergent patterns related to requirements and projects.

4. Future investigations can enhance the interface among applications, performing a deeper analysis concerning the API built.

5. Future studies may perform a wider comparative analysis with related works to investigate the results obtained in this study, exploring the data used and the saved time through the use of Nhatos.

\section{ACKNOWLEDGEMENTS}

We would like to thank University of Vale do Rio dos Sinos (UNISINOS), VALORIZA, and COPELABS for embracing this research.

\section{FUNDING}

This work was supported by national funds through the Fundação para a Ciência e a Tecnologia, I.P. (Portuguese Foundation for Science and Technology) by the project UIDB/05064/2020 (VALORIZA—Research Centre for Endogenous Resource Valorization), and Project UIDB/04111/2020, ILIND-Instituto Lusófono de Investigação e Desenvolvimento, under project COFAC/ILIND/COPELABS/3/2020.

\section{REFERENCES}

Abech, M., da Costa, C. A., Barbosa, J. L. V., Rigo, S. J., and da Rosa Righi, R. (2016). A model for learning objects adaptation in light of mobile and context-aware computing. Personal and Ubiquitous Computing, 20(2):167-184. DOI 10.1007/s00779-016-0902-3.

Aiken, M. (2019). An Updated Evaluation of Google Translate Accuracy. Studies in Linguistics and Literature, 3(3):253. DOI 10.22158/sll.v3n3p253.

Aranda, J. A. S., Bavaresco, R. S., de Carvalho, J. V., Yamin, A. C., Tavares, M. C., and Barbosa, J. L. V. (2021). A computational model for adaptive recording of vital signs through context histories. Journal of Ambient Intelligence and Humanized Computing, pages 1-15. DOI 10.1007/s12652-021-03126-8. 
Bakar, N. H., Kasirun, Z. M., Salleh, N., and Jalab, H. A. (2016). Extracting features from online software reviews to aid requirements reuse. Applied Soft Computing, 49:1297-1315. DOI 10.1016/j.asoc.2016.07.048.

Barbosa, J., Tavares, J., Cardoso, I., Alves, B., and Martini, B. (2018). Trailcare: An indoor and outdoor context-aware system to assist wheelchair users. International Journal of Human-Computer Studies, 116:1 - 14. DOI 10.1016/j.ijhcs.2018.04.001.

Barbosa, J. L. V., Barbosa, D. N. F., de Oliveira, J. M., and Jr., S. A. R. (2014). A decentralized infrastructure for ubiquitous learning environments. Journal of Universal Computer Science, 20(12):1649-1669. DOI 10.3217/jucs-020-12-1649.

Barbosa, J. L. V., Hahn, R. M., Barbosa, D. N. F., and Saccol, A. I. d. C. Z. (2011). A ubiquitous learning model focused on learner interaction. International Journal of Learning Technology, 6(1):62-83. DOI 10.1504/IJLT.2011.040150.

Barbosa, J. L. V., Martins, C., Franco, L. K., and Barbosa, D. N. F. (2016). Trailtrade: A model for trail-aware commerce support. Computers in Industry, 80:43-53. DOI 10.1016/j.compind.2016.04.006.

Barbosa, J. L. V., Sempe, B. K., Mota, B., and Dini, L. I. (2017). An anesthesia alert system based on dynamic profiles inferred through the medical history of patients. J. Univers. Comput. Sci., 23(8):705724. DOI 10.3217/jucs-023-08-0705.

Bavaresco, R., Barbosa, J., Vianna, H., Büttenbender, P., and Dias, L. (2020). Design and evaluation of a context-aware model based on psychophysiology. Computer Methods and Programs in Biomedicine, 189:1-14. DOI 10.1016/j.cmpb.2019.105299.

Bhanu, K., Reddy, T. B., and Hanumanthappa, M. (2019). Multi-agent based context aware information gathering for agriculture using wireless multimedia sensor networks. Egyptian Informatics Journal, 20(1):33-44. DOI 10.1016/j.eij.2018.07.001.

Bozyiğit, F., Özlem Aktaş, and Kılınç, D. (2021). Linking software requirements and conceptual models: A systematic literature review. Engineering Science and Technology, an International Journal, 24(1):71-82. DOI 10.1016/j.jestch.2020.11.006.

Da Rosa, J. H., Barbosa, J. L., and Ribeiro, G. D. (2016). ORACON: An adaptive model for context prediction. Expert Systems with Applications, 45:56-70. DOI 10.1016/j.eswa.2015.09.016.

Dalmina, L., Barbosa, J. L. V., and Vianna, H. D. (2019). A systematic mapping study of gamification models oriented to motivational characteristics. Behaviour and Information Technology, 38(11):11671184. DOI 10.1080/0144929X.2019.1576768.

de Souza, R. S., Barbará Lopes, J. L., Resin Geyer, C. F., da Rosa Silveira João, L., Afonso Cardozo, A., Corrêa Yamin, A., Gadotti, G. I., and Victoria Barbosa, J. L. (2019). Continuous monitoring seed testing equipaments using internet of things. Computers and Electronics in Agriculture, 158:122-132. DOI 10.1016/j.compag.2019.01.024.

Dey, A. K., Abowd, G. D., and Salber, D. (2001). A conceptual framework and a toolkit for supporting the rapid prototyping of context-aware applications. Hum.-Comput. Interact., 16(2):97-166. DOI 10.1207/S15327051HCI16234_02.

Dias, L. P. S., Barbosa, J. L. V., Feijó, L. P., and Vianna, H. D. (2020). Development and testing of iaware model for ubiquitous care of patients with symptoms of stress, anxiety and depression. Computer Methods and Programs in Biomedicine, 187:105113. DOI 10.1016/j.cmpb.2019.105113.

Dick, J., Hull, E., and Jackson, K. (2017). Requirements Engineering, volume 4. Springer, Newtown Square, PA. DOI 10.1007/978-3-319-61073-3.

Dupont, D., Barbosa, J. L. V., and Alves, B. M. (2020). CHSPAM: a multi-domain model for sequential pattern discovery and monitoring in contexts histories. Pattern Analysis and Applications, 23(2):725734. DOI 10.1007/s10044-019-00829-9.

D'Avila, L. F., Barbosa, J. L. V., and de Oliveira, K. F. (2020a). Sw-context: a model to improve developers' situational awareness. IET Software, 14(5):535-543. DOI 10.1049/iet-sen.2018.5156.

D'Avila, L. F., de Oliveira, K. F., and Barbosa, J. L. V. (2020b). Effects of contextual information on maintenance effort: A controlled experiment. Journal of Systems and Software, 159:1-19. DOI 10.1016/j.jss.2019.110443.

Ferreira, L. G., Barbosa, J. L. V., Gluz, J. C., Matter, V. K., and Barbosa, D. N. F. (2020). Using Learner Group Profiles for Content Recommendation in Ubiquitous Environments: International Journal of Information and Communication Technology Education, 16(4):1-19. DOI 10.4018/IJICTE.2020100101. 
Filippetto, A. S., Barcelos, G., Barbosa, J. L. V., Francisco, R., and Saccol, A. I. C. Z. (2020). A ubiquitous project management model based on context. International Journal of Business Information Systems (Print), 1:1-25. DOI 10.1504/IJBIS.2020.10023985.

Filippetto, A. S., Lima, R., and Barbosa, J. L. V. (2021). A risk prediction model for software project management based on similarity analysis of context histories. Information and Software Technology, 131:1-37. DOI 10.1016/j.infsof.2020.106497.

Garcia, J. E. and Paiva, A. C. R. (2016). REQAnalytics: A recommender system for requirements maintenance. International Journal of Software Engineering and Its Applications, 10(1):129-140. DOI 10.14257/ijseia.2016.10.1.13.

Google (2021a). Cloud natural language derive insights from unstructured text using google machine learning. https://cloud.google.com/natural-language [Online; Accessed 2021-06-18].

Google (2021b). Cloud translation dynamically translate between languages. https://cloud.google.com/translate [Online; Accessed 2021-06-18].

Hastie, S. and Wojewoda, S. (2015). Standish Group Chaos Report - QA with Jennifer Lynch. https://www.infoq.com/articles/standish-chaos-2015 [Online; Accessed 2021-06-18].

Helfer, G. A., Victória Barbosa, J. L., dos Santos, R., and da Costa, A. B. (2020). A computational model for soil fertility prediction in ubiquitous agriculture. Computers and Electronics in Agriculture, 175. DOI 10.1016/j.compag.2020.105602.

Hujainah, F., Binti Abu Bakar, R., Nasser, A. B., Al-haimi, B., and Zamli, K. Z. (2021). Srptackle: A semi-automated requirements prioritisation technique for scalable requirements of software system projects. Information and Software Technology, 131. DOI 10.1016/j.infsof.2020.106501.

Irshad, M., Petersen, K., and Poulding, S. (2018). A systematic literature review of software requirements reuse approaches. Information and Software Technology, 93:223-245. DOI 10.1016/j.infsof.2017.09.009.

Kim, M., Dey, S., and Lee, S.-W. (2019). Ontology-driven security requirements recommendation for apt attack. In 2019 IEEE 27th International Requirements Engineering Conference Workshops (REW), pages 150-156. DOI 10.1109/REW.2019.00032.

Kusner, M. J., Sun, Y., Kolkin, N. I., and Weinberger, K. Q. (2015). From word embeddings to document distances. Proceedings of the 32nd International Conference on International Conference on Machine Learning, 37:957-966.

Larentis, A. V., Barbosa, D. N. F., da Silva, C. R., and Barbosa, J. L. V. (2020). Applied Computing to Education on Noncommunicable Chronic Diseases: A Systematic Mapping Study. Telemedicine journal and e-health : the official journal of the American Telemedicine Association, 26(2):147-163. DOI 10.1089/tmj.2018.0282.

Larioui, J. (2020). Multi-agent system architecture oriented prometheus methodology design for multimodal transportation. International Journal of Emerging Trends in Engineering Research, 8:2118-2125. DOI 10.30534/ijeter/2020/105852020.

Leithardt, V., Correia, L., Borges, G., Rossetto, A., Rolim, C., Geyer, C., and Sá Silva, J. (2018). Mechanism for privacy management based on data history (ubipri-his). Journal of Ubiquitous Systems and Pervasive Networks, 10:11-19. DOI 10.5383/JUSPN.10.01.002.

Leithardt, V., Santos, D., Silva, L., Viel, F., Zeferino, C., and Silva, J. (2020). A solution for dynamic management of user profiles in iot environments. IEEE Latin America Transactions, 18(07):1193-1199. DOI 10.1109/TLA.2020.9099759.

Liu, X., Leng, Y., Yang, W., Zhai, C., and Xie, T. (2018). Mining android app descriptions for permission requirements recommendation. In 2018 IEEE 26th International Requirements Engineering Conference (RE), pages 147-158. DOI 10.1109/RE.2018.00024.

Lopes, J., Souza, R., Geyer, C., Costa, C., Barbosa, J. L. V., Pernas, A., and Yamin, A. (2014). A middleware architecture for dynamic adaptation in ubiquitous computing. Journal of Universal Computer Science, 20(9):1327-1351. DOI 10.3217/jucs-020-09-1327.

Luisa, M., Mariangela, F., and Pierluigi, N. (2004). Market research for requirements analysis using linguistic tools. Requirements Engineering, 9(1):40-56. DOI 10.1007/s00766-003-0179-8.

Machado, S. D., Tavares, J. E. d. R., Martins, M. G., Barbosa, J. L. V., González, G. V., and Leithardt, V. R. Q. (2021). Ambient intelligence based on iot for assisting people with alzheimer's disease through context histories. Electronics, 10(11). DOI 10.3390/electronics10111260.

Martini, B. G., Helfer, G. A., Barbosa, J. L. V., Espinosa Modolo, R. C., da Silva, M. R., de Figueiredo, 
R. M., Mendes, A. S., Silva, L. A., and Leithardt, V. R. Q. (2021). Indoorplant: A model for intelligent services in indoor agriculture based on context histories. Sensors, 21(5). DOI 10.3390/s21051631.

Matos, C. M., Matter, V. K., Martins, M. G., da Rosa Tavares, J. E., Wolf, A. S., Buttenbender, P. C., and Barbosa, J. L. V. (2021). Towards a collaborative model to assist people with disabilities and the elderly people in smart assistive cities. Journal of Universal Computer Science, 27(1):65-86. DOI $10.3897 /$ jucs.64591.

Mikolov, T., Chen, K., Corrado, G., and Dean, J. (2013). Efficient estimation of word representations in vector space. arXiv:1301.3781.

Mougouei, D. and Powers, D. M. (2021). Dependency-aware software requirements selection using fuzzy graphs and integer programming. Expert Systems with Applications, 167. DOI 10.1016/j.eswa.2020.113748.

Nadkarni, S. and Prügl, R. (2021). Digital transformation: a review, synthesis and opportunities for future research. Management Review Quarterly, 71(2):233-341. DOI 10.1007/s11301-020-00185-7.

Orrego, R. B. S. and Barbosa, J. L. V. (2019). A model for resource management in smart cities based on crowdsourcing and gamification. Journal of Universal Computer Science, 25(8):1018-1038. DOI 10.3217/jucs-025-08-1018.

Padgham, L. and Winikoff, M. (2004). Developing intelligent agent systems: A practical guide. Journal. DOI 10.1002/0470861223.

Petry, M. M., Barbosa, J. L. V., Rigo, S. J., Dias, L. P. S., and Büttenbender, P. C. (2020). Toward a ubiquitous model to assist the treatment of people with depression. Universal Access in the Information Society, 19(4):841-854. DOI 10.1007/s10209-019-00697-4.

Portugal, R. L. Q., Casanova, M. A., Li, T., and do Prado Leite, J. C. S. (2017). GH4RE: repository recommendation on github for requirements elicitation reuse. In Proceedings of the Forum and Doctoral Consortium Papers Presented at the 29th International Conference on Advanced Information Systems Engineering, CAiSE 2017, Essen, Germany, June 12-16, 2017, pages 113-120.

Project Management Institute (2017a). A Guide to the Project Management Body of Knowledge

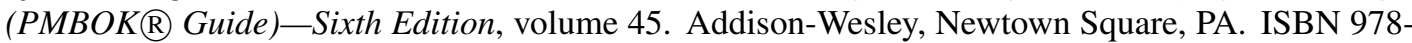
1628251845.

Project Management Institute (2017b). Requirements Management: A practice Guide, volume 1. Project Management Institute.

Robillard, M., Maalej, W., Walker, R., and Zimmermann, T. (2014). Recommendation Systems in Software Engineering, volume 1. Springer. DOI 10.1007/978-3-642-45135-5.

Rolim, C. O., Rossetto, A. G., Leithardt, V. R., Borges, G. A., Geyer, C. F., dos Santos, T. F., and Souza, A. M. (2016). Situation awareness and computational intelligence in opportunistic networks to support the data transmission of urban sensing applications. Computer Networks, 111:55-70. DOI 10.1016/j.comnet.2016.07.014.

Rosa, J. H., Barbosa, J. L., Kich, M., and Brito, L. (2015). A Multi-Temporal Context-aware System for Competences Management. International Journal of Artificial Intelligence in Education, 25(4):455-492. DOI 10.1007/s40593-015-0047-y.

SAP (2021). TAM - The SAP way combining FMC and UML. http://www.fmc-modeling.org/fmc-and-tam [Online; Accessed 2021-06-18].

Satyanarayanan, M. (2001). Pervasive computing: vision and challenges. IEEE Personal Communications, 8(4):10-17. DOI 10.1109/98.943998.

Silva, J. M., Rosa, J. H., Barbosa, J. L. V., Barbosa, D. N. F., and Palazzo, L. A. M. (2010). Content distribution in trail-aware environments. Journal of the Brazilian Computer Society, 16(3):163-176. DOI 10.1007/s13173-010-0015-1.

Silver, G. A. (2014). The use of ontologies in discrete-event simulation. Global Journal of Researches in Engineering, 8.

Sutherland, J. and Coplien, J. O. (2019). A Scrum Book - The Spirit of the Game, volume 2. Pragmatic Bookshelf. ISBN 9781680506716.

Swathine, K. and Sumathi, N. (2021). An adaptive optimization based meta-heuristic approach for tracing software requirements. Materials Today: Proceedings. DOI 10.1016/j.matpr.2021.01.462.

Tavares, J., Barbosa, J., Cardoso, I., Costa, C., Yamin, A., and Real, R. (2016). Hefestos: an intelligent system applied to ubiquitous accessibility. Universal Access in the Information Society, 15(4):589-607. DOI 10.1007/s10209-015-0423-2. 
Vianna, H. D. and Barbosa, J. (2014). A model for ubiquitous care of noncommunicable diseases. IEEE Journal of Biomedical and Health Informatics, 18(5):1597-1606. DOI 10.1109/JBHI.2013.2292860.

Vianna, H. D. and Barbosa, J. L. V. (2019). A scalable model for building context-aware applications for noncommunicable diseases prevention. Information Processing Letters, 148:1-6. DOI 10.1016/j.ipl.2019.03.010.

Vianna, H. D., Victória Barbosa, J. L. V., and Pittoli, F. (2017). In the pursuit of hygge software. IEEE Software, 34(6):48-52. DOI 10.1109/MS.2017.4121208.

Villela, K., Groen, E. C., and Doerr, J. (2019). Ubiquitous requirements engineering: A paradigm shift that affects everyone. IEEE Software, 36(2):8-12. DOI 10.1109/MS.2018.2883876.

Villela, K., Hess, A., Koch, M., Falcao, R., Groen, E. C., Dörr, J., Valero, C. N., and Ebert, A. (2018). Towards ubiquitous RE: A perspective on requirements engineering in the era of digital transformation. In 2018 IEEE 26th International Requirements Engineering Conference (RE), pages 205-216. DOI 10.1109/RE.2018.00029.

Wagner, A., Barbosa, J. L. V., and Barbosa, D. N. F. (2014). A model for profile management applied to ubiquitous learning environments. Expert Systems with Applications, 41(4):2023-2034. DOI 10.1016/j.eswa.2013.08.098.

Weiser, M. (1999). The computer for the 21 st century . ACM SIGMOBILE Mobile Computing and Communications Review, 3(3):3-11. DOI 10.1145/329124.329126.

Wiedmann, T., Barbosa, J. L. V., Rigo, S. J., and Barbosa, D. N. F. (2016). Recsim: A model for learning objects recommendation using similarity of sessions. Journal of Universal Computer Science, 22(8):1175-1200. DOI 10.3217/jucs-022-08-1175.

Williams, G. and Mahmoud, A. (2017). Mining Twitter feeds for software user requirements. 2017 IEEE 25th International Requirements Engineering Conference (RE), pages 1-10. DOI 10.1109/RE.2017.14. Xie, H., Yang, J., Chang, C. K., and Liu, L. (2017). A statistical analysis approach to predict user's changing requirements for software service evolution. Journal of Systems and Software, 132:147-164. DOI 10.1016/j.jss.2017.06.071. 